Draft version March 7, 2018

Preprint typeset using $\mathrm{LATEX}_{\mathrm{E}}$ style emulateapj v. 6/22/04

\title{
CENTRIFUGAL BREAKOUT OF MAGNETICALLY CONFINED LINE-DRIVEN STELLAR WINDS
}

\author{
Asif ud-Doula*, Richard H. D. Townsend, and Stanley P. Owocki \\ Bartol Research Institute, Department of Physics \& Astronomy, University of Delaware, Newark, DE 19716 \\ Draft version March 7, 2018
}

\begin{abstract}
We present 2D MHD simulations of the radiatively driven outflow from a rotating hot star with a dipole magnetic field aligned with the star's rotation axis. We focus primarily on a model with moderately rapid rotation (half the critical value), and also a large magnetic confinement parameter, $\eta_{*} \equiv B_{*}^{2} R_{*}^{2} / \dot{M} V_{\infty}=600$. The magnetic field channels and torques the wind outflow into an equatorial, rigidly rotating disk extending from near the Kepler corotation radius outwards. Even with fine-tuning at lower magnetic confinement, none of the MHD models produce a stable Keplerian disk. Instead, material below the Kepler radius falls back on to the stellar surface, while the strong centrifugal force on material beyond the corotation escape radius stretches the magnetic loops outwards, leading to episodic breakout of mass when the field reconnects. The associated dissipation of magnetic energy heats material to temperatures of nearly $10^{8} \mathrm{~K}$, high enough to emit hard (several keV) X-rays. Such centrifugal mass ejection represents a novel mechanism for driving magnetic reconnection, and seems a very promising basis for modeling X-ray flares recently observed in rotating magnetic Bp stars like $\sigma$ Ori E.

Subject headings: MHD — stars: winds — stars: magnetic fields — stars: rotation — stars: flare X-rays: stars
\end{abstract}

\section{INTRODUCTION}

Magnetic fields can greatly influence stellar winds by guiding the outflowing plasma along field lines (e.g., MacGregor 1988; Shore \& Brown 1990). The extent of this influence depends largely on the magnetic field strength and the wind mass loss rate. Two-dimensional (2D) magnetohydrodynamical (MHD) simulations of axisymmetric hot-star winds with no rotation show that the competition between the wind and the field can be quite well characterized by a single wind magnetic confinement parameter, $\eta_{*}$, as defined by ud-Doula \& Owocki (2002), and described further in 2 below.

For significant confinement (i.e., $\eta_{*} \gtrsim 10$ ), material driven from opposite footpoints of closed magnetic loops is forced to collide near the loop tops, leading to a Magnetically Confined Wind Shock (MCWS) scenario for production of intermediate-hardness X-rays (Babel \& Montmerle 1997a b). For the magnetic hot star $\theta^{1}$ Ori $\mathrm{C}$, which is inferred to have both a moderately strong wind (with mass loss rate $\approx 10^{-7} M_{\odot} /$ yr) and a large-scale, dipole field of strength $B_{*} \approx 1100 \mathrm{G}$ (Donati et al. 2002), recent MHD simulations based on this MCWS paradigm provide quite good agreement with the X-ray spectrum observed by Chandra (Gagné et al. $2005)$. Indeed, because $\theta^{1}$ Ori $\mathrm{C}$ is a relatively slow rotator (with period $\sim 15$ days), even the observed rotational modulation can be well accounted for by simply varying the observational perspective within a non-rotating dynamical simulation model.

The purpose of this letter is to report on initial MHD simulation results for more rapidly rotating hot stars, in which the rotation can have a direct dynamical effect on the overall evolution of the wind outflow and its confinement. The results have particular rele-

\footnotetext{
* also at Department of Physics, Swarthmore College, Swarthmore, PA 19081
}

vance in the context of two proposed models for rotating, magnetic hot stars, known generally as the $\mathrm{Mag}$ netically Torqued Disk (MTD) model (Cassinelli et al. 2002) and the Rigidly Rotating Magnetosphere (RRM) model (Townsend \& Owocki 2005). The MTD has been proposed as a scenario for producing Keplerian decretion disks, such as inferred from the Balmer line emission of classical Be stars. The RRM leads instead to rigid-body disks or clouds that provide a promising way to explain the rotationally modulated Balmer emission from strongly magnetic $\mathrm{Ap} / \mathrm{Bp}$ stars like the $\mathrm{B} 2 \mathrm{pV}$ star $\sigma$ Ori E (Townsend et al. 2005).

After first casting the relative importance of magnetic confinement vs. rotation in terms of characteristic Alfvèn vs. Kepler corotation radii (\$2), we show in $\$ 3$ that even for parameters fine-tuned to optimize the chances of a producing a Keplerian disk, our 2D MHD simulations for rotationally aligned, dipole fields instead produce a combination of inflow and outflow for material below and above the Kepler corotation radius, and never lead to the kind of extended Keplerian disk envisioned in the MTD scenario.

On the other hand, we show in that a model with strong magnetic confinement $\left(\eta_{*}=600\right)$ does lead to a rigid-body disk, much as predicted in the RRM model. However, the outer regions of this disk are characterized by episodic breakout of material, driven by the strong net outward centrifugal force associated with the spin-up of material into corotation with the underlying star. Moreover, the energy release associated with magnetic reconnection in these breakout events heats material to extremely high temperatures, providing a promising mechanism for explaining the very hard X-ray flare emission recently observed from magnetic Bp stars such as $\sigma$ Ori E. We conclude in $\$ 5$ with a brief summary of our findings, noting also the need for further work on the detailed physics of this somewhat-novel, centrifugally 
driven reconnection mechanism.

\section{ALFVÈN VS. KEPLER COROTATION RADII}

MHD simulations ud-Doula \& Owocki 2002; ud-Doula 2003) indicate that the effectiveness of magnetic fields in channeling a stellar wind outflow can be characterized by the ratio between the magnetic and wind energy densities

$$
\eta(r) \equiv \frac{B^{2} / 8 \pi}{\rho v^{2} / 2}=\eta_{*} \frac{\left(r / R_{*}\right)^{2-2 q}}{\left(1-R_{*} / r\right)^{\beta}} .
$$

This ratio furnishes an indication of whether the magnetic field at radius $r$ dominates the wind outflow $(\eta \gg$ $1)$, or vice versa $(\eta \ll 1)$. As indicated by the second equality, its overall scaling is set by the "magnetic confinement parameter" $\eta_{*} \equiv B_{*}^{2} R_{*}^{2} / \dot{M} V_{\infty}$, which depends on the strength of the field $B_{*}$ at the stellar surface radius $r=R_{*}$, and on the wind terminal momentum $\dot{M} V_{\infty}$. The radial variation of $\eta$ is then modeled in terms of a magnetic power-law index $q$ (=3 for a dipole) and a velocity index $\beta$ ( $\approx 1$ for a standard CAK wind). If for simplicity we ignore the wind velocity variation (i.e. by taking $\beta=0$ ), the Alfvèn radius - at which the magnetic and wind energy densities are equal, $\eta\left(R_{\mathrm{A}}\right) \equiv 1-$ can be solved explicitly as

$$
R_{\mathrm{A}}=\eta_{*}^{1 / 4} R_{*} .
$$

As shown by simulation results summarized in ud-Doula \& Owocki (2002), this Alfvèn radius provides a reasonable estimate for the maximum radius of magnetic loops that remain closed in a wind outflow. Moreover, since in rotating models such closed loops tend to keep the outflow in rigid-body rotation with the underlying star, it also defines the radius of maximum rotational spin-up of the wind azimuthal velocity $v_{\phi}$.

To characterize such rotational effects, let us next define a Kepler corotation radius $R_{\mathrm{K}}$. Analogous to the terrestrial geostationary orbital radius, $R_{\mathrm{K}}$ is the point at which the centrifugal force arising due to corotation balances the local gravitational force from the star. That is,

$$
\frac{G M_{*}}{R_{\mathrm{K}}^{2}}=\frac{v_{\phi}^{2}}{R_{\mathrm{K}}}=\frac{V_{\mathrm{eq}}^{2}}{R_{*}^{2}},
$$

where $V_{\text {eq }}$ is the equatorial rotation velocity of the star. Solving this equation, we find the Kepler radius as

$$
R_{\mathrm{K}}=W^{-2 / 3} R_{*},
$$

where $W \equiv V_{\text {eq }} / V_{\text {crit }}$ is ratio of the star's equatorial velocity to the critical velocity $V_{\text {crit }} \equiv \sqrt{G M_{*} / R_{*}}$.

Finally, it is also worth noting here that corotation out to an only slightly higher escape radius,

$$
R_{\mathrm{E}}=2^{1 / 3} R_{\mathrm{K}}=2^{1 / 3} W^{-2 / 3} R_{*},
$$

would imply an azimuthal velocity $v_{\phi}$ that equals the local escape speed from the star's gravitational field.

\section{ATTEMPTS TO FINE TUNE FOR A KEPLERIAN DISK}

The above scalings suggest that a likely necessary condition for propelling outflowing material into a Keplerian disk (as envisioned in the MTD scenario for classical Be stars) is to choose a combination of parameters

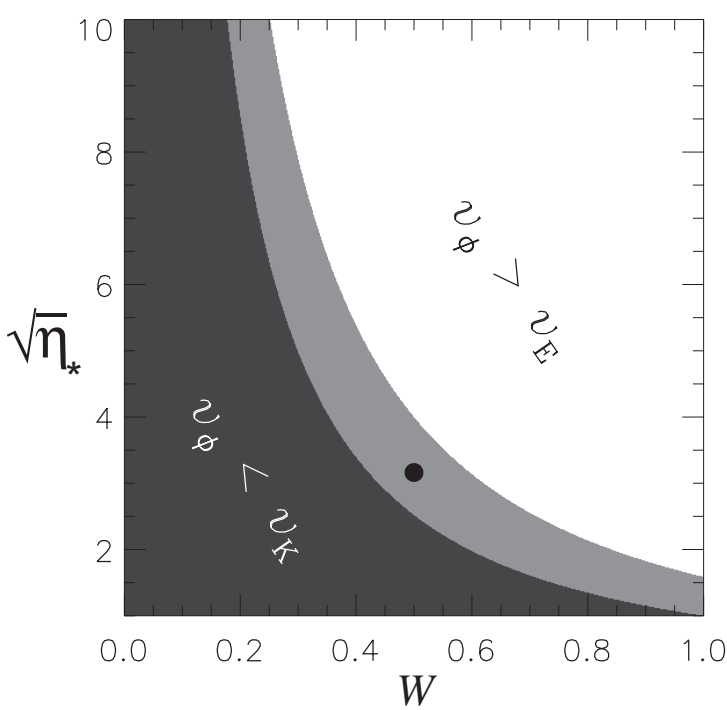

FIG. 1. - The key domains in a parameter plane of magnetic field strength (as represented by $\sqrt{\eta_{*}} \sim B_{*}$ ) vs. stellar rotation (as represented by the critical rotation fraction $W \sim V_{\text {eq }}$ ). The black area to the lower left denotes models in which the magnetic spin-up of the azimuthal velocity $v_{\phi}$ is insufficient to reach the velocity $v_{\mathrm{K}}$ required for Keplerian orbit, while the white area in the upper right denotes models in which the spin-up leads to $v_{\phi}$ that exceed the escape speed $v_{\mathrm{E}}$. Only in the gray area, bounded between curves for models defined by $R_{\mathrm{A}}=R_{\mathrm{K}}$ and $R_{\mathrm{A}}=R_{\mathrm{E}}$, does spin-up into Keplerian orbit seem possible. The dot positioned in the middle of this area indicates the parameters of the MHD simulations described in $\$ 3$

for magnetic confinement vs. stellar rotation such that $R_{\mathrm{K}}<R_{\mathrm{A}}<R_{\mathrm{E}}$. In the parameter plane of $\sqrt{\eta_{*}} \sim B_{*}$ vs. $W \sim V_{\text {eq }}$ illustrated in Fig. 1] the required combination is represented by the relatively narrow gray band.

To obtain a model that has the best chance of yielding a Keplerian disk, we therefore conduct $2 \mathrm{D}$ axisymmetric MHD simulations of a star having parameters $\eta_{*}=10$ and $W=0.5$ that fall in the middle of the gray area in Fig. 1. The initial magnetic field geometry is assumed to be a dipole aligned with the rotation axis. Calculations are undertaken using the publicly available ZEUS-3D code (Stone \& Norman 1992), augmented to include radiative driving and radiative cooling (Gagné et al. 2005), with typical early B-star values for the stellar radius and mass, $R_{*}=6.4 R_{\odot}$ and $M_{*}=11 M_{\odot}$. The numerical grid and boundary conditions follow those given in ud-Doula \& Owocki (2002). Since the star is rotating at one half its critical value, the oblateness and gravity darkening of the stellar surface are relatively small, and are neglected in our simulations.

Figure 2 illustrates results from the simulation. The left-hand panel shows the logarithm of density (superimposed with field lines) at a time $90 \mathrm{ks}$ after the introduction of the dipole field into a spherically symmetric wind. Superficially, these conditions do temporarily resemble the MTD scenario. However, a closer inspection shows that most of the density-enhanced equatorial region does not have the appropriate $v_{\phi}$ necessary for a stable, stationary, Keplerian orbit. Indeed, in just a few ks of subsequent evolution this putative "disk" becomes completely disrupted, characterized generally by infall of the material in the inner region, i.e. below the Kepler 


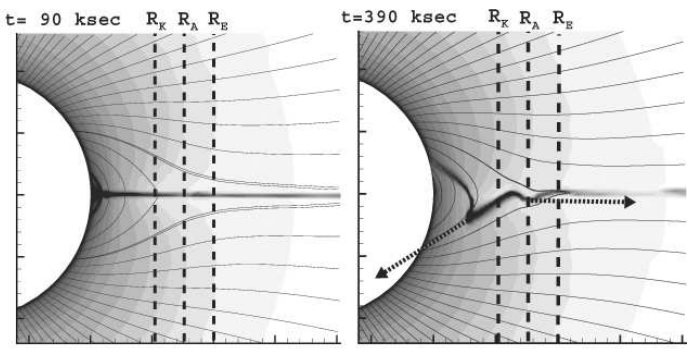

FIG. 2.- Density in a 2D MHD simulation with $\eta_{*}=10$ and $W=1 / 2$, shown at time snapshots of $90 \mathrm{ks}$ (left) and $390 \mathrm{ks}$ (right) after a dipole field is introduced into an initially steady, spherical, unmagnetized, line-driven stellar wind. The curves denote magnetic field lines, and the vertical dashed lines indicate the equatorial location of the Kepler $\left(R_{\mathrm{K}}\right)$, Alfvèn $\left(R_{\mathrm{A}}\right)$, and escape $\left(R_{\mathrm{E}}\right)$ radii defined in eqns. (4), 2], and (5), respectively The arrows denote the upward and downward flow above and below the Kepler radius, emphasizing that the material never forms a stable, orbiting disk.

radius $R_{\mathrm{K}}$, and by outflow in the outer region above the Kepler radius. The right-hand panel demonstrates this by showing the irregular form of the dense compression at an arbitrarily chosen later time $(390 \mathrm{ks})$. The bold arrows emphasize the flow divergence of the dense material both downward and upward from the Kepler radius. (The overall evolution is most vividly illustrated through animations available on the internet $^{2}$.)

We have carried out a number of other similar MHD simulations exploring parameter space in both the rotation rate and magnetic field strength directions. In every case, we find that the equatorial compressions are dominated by similar radial inflows and/or outflows, with no stable Keplerian disks forming. We thus conclude that stationary, large-scale magnetic fields are dynamically ill-suited for producing Keplerian or near-Keplerian decretion disks.

\section{A CENTRIFUGAL BREAKOUT MECHANISM FOR} X-RAY FLARING

A recent study by Townsend \& Owocki (2005) suggests that, in the limit of very strong magnetic confinement $\left(\eta_{*} \gg 1\right)$, magnetic torquing can readily produce rigid-body disks. In this strong-field limit, the field lines can be idealized as rigid pipes that channel the wind outflow and force it to corotate with the underlying star. This leads to a rigidly rotating magnetosphere in which material accumulates near minima of the effective gravito-centrifugal potential along each field line. Townsend et al. (2005) show that the resulting circumstellar clouds provide a natural explanation for the observed rotational modulation of both the photometric continuum and Balmer line emission in the $\mathrm{B} 2 \mathrm{pV}$ star $\sigma$ Ori E.

A key feature of this RRM model is that the magnetic field not only torques up outflowing material into corotation around the star, but then also holds it down against the net outward centrifugal acceleration beyond the Kepler radius. In the semi-analytic RRM analysis, the field is idealized as being strong enough to enforce corotation and confinement at arbitrarily large radii, and for arbitrarily long times. More realistically, however, for large but finite field strengths, the secular accumulation of ma- terial into the RRM means that these idealizations must break down once the total outward centrifugal force exceeds a level that can be balanced by the finite magnetic tension.

Townsend \& Owocki (2005) speculate that this will lead to centrifugal breakout of material, and give estimates for the expected characteristic breakout timescale as a function of distance above the Kepler radius (see their Appendices A and B). For the case of $\sigma$ Ori E, they estimate that the largest-scale evacuations can be expected every $\sim 10-100 \mathrm{yr}$, but they also anticipate a whole hierarchy of breakout events extending down to timescales of days.

Our present MHD simulations allow us to examine the dynamics of this breakout process. Unfortunately, at the confinement parameter $\eta_{*} \sim 10^{7}$ appropriate to $\sigma$ Ori E, the Alfvèn speed becomes very large, implying a very small Courant time step to ensure numerical stability; this together with the full 3D geometry needed to model the oblique-field tilt relative to the rotation axis makes direct MHD simulation of the $\sigma$ Ori E case prohibitively expensive. Instead, we consider the more tractable, 2D axisymmetric case of a field-aligned dipole with only moderately strong magnetic confinement $\left(\eta_{*}=600\right)$. As appropriate for $\sigma$ Ori E, we again set the rotation to half the critical rate $(W=0.5)$, with other stellar and grid parameters also the same as used in $\$ 3$.

Snapshots from the simulations, plotted in Fig. 3] reveal that there forms a rigidly rotating disk near and above the Kepler radius $R_{\mathrm{K}} / R_{*} \approx 0.5^{-2 / 3} \approx 1.6$, as predicted by the RRM model. But in the outer regions of this disk (near the Alfvèn radius $R_{\mathrm{A}} / R_{*} \approx 600^{1 / 4} \approx 5$ ), there is indeed also a semi-regular sequence of breakout events. During a breakout, the magnetic field lines become so drawn out by the ejected plasma that they reconnect, snapping back toward the star (cf. middle and right panels of Fig. 31). The energy release associated with this reconnection, and its subsequent dissipation via radiative cooling, represents a strong candidate for producing the hard X-ray flares observed in $\sigma$ Ori E by ROSAT (Groote \& Schmitt 2004) and XMM-Newton (Sanz-Forcada et al. 2004).

This scenario is reminiscent of fast magnetic reconnection processes invoked for solar flares (e.g., Petschek 1966; Nitta 2004). But in the present case, the work to stretch the magnetic fields comes from the rotational energy of the dense plasma at the edge of the disk, with the trigger for reconnection being the wind impingent at an oblique angle from higher latitudes. The centrifugal energy stored temporarily in the magnetic field is thereby converted into thermal energy, heating nearby material to temperatures $T \sim 10^{8} \mathrm{~K}$, high enough to produce the hard $(\geq 2 \mathrm{keV})$ components of the X-ray flares observed in $\sigma$ Ori E. A similar mechanism may also be relevant to X-ray outbursts recently observed in other (possibly magnetic) early-type stars, such as the mid-B star HD 38563S (Yanagida et al. 2004) and the late-O star $\theta^{2}$ Ori A (Feigelson et al. 2002).

The centrifugal breakout here provides an interesting counterexample to the "slingshot prominence" model of Jardine \& van Ballegooijen (2005). This coolstar, coronal model envisions a tearing-mode instability (Furth et al. 1963) in the current sheet dividing open

http://www.bartol.udel.edu/ owocki/animations/den4wp5eta10.avi 

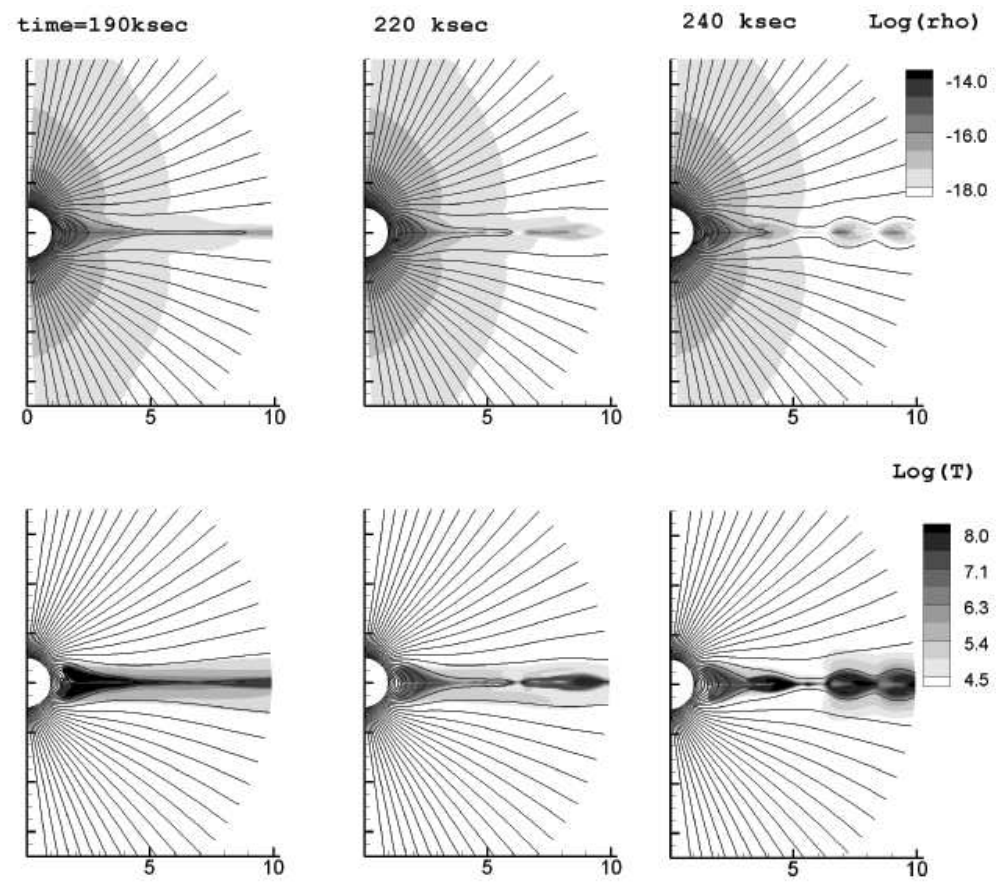

FIG. 3.- MHD simulation results for a radiatively driven stellar wind from a star rotating at half the critical rate, with a rotationaligned dipole field that has a magnetic confinement parameter $\eta_{*}=600$. The grayscales show the logarithm of density (top panels) and temperature (bottom panels) at three sample time snapshots, chosen to show the centrifugal ejection of material accumulated in an equatorial disk. The associated stretching and eventual reconnection of the field lines heats material to temperatures of order $10^{8} \mathrm{~K}$, hot enough to emit hard (few $\mathrm{keV}$ ) X-rays.

field regions in an overlying, gas-pressure-driven wind. This leads to a magnetic reconnection that effectively traps some of the outflowing wind material, contributing then to an extended, quasi-static coronal prominence (Jardine et al. 2001). Although the centrifugal support of this prominence is similar to our RRM scenario for hotstars, in this cool-star case the high coronal back-pressure chokes off any footpoint upflow that could continuously feed the accumulation region above the Kepler radius. In our hot-star model, it is this secular accumulation of material that eventually forces centrifugal breakout and drives the associated reconnection. As such, unlike the tearing-mode reconnection postulated in this cool-star context, we believe the overall rate of reconnection and mass ejection should be relatively independent of the specific instabilities and plasma processes at play.

In real plasmas, the details for magnetic reconnection can be quite complex, involving non-thermal particle acceleration (Wiechen et al. 1995) and/or high-speed jets that form dissipative shocks (Petschek 1966), with the distribution of heat modified by thermal conduction or non-local particle transport (Mivagoshi \& Yokovama 2003). The Zeus-3D numerical code used here is based on ideal an MHD formalism that effectively ignores these complexities, and indeed does not even include any explicit resistivity that could heat material via Ohmic dissipation. Instead, magnetic reconnection is enabled by the finite spatial grid resolution, leading to grid-scale currents inducing high-speed jets that quickly dissipate the flow energy in localized shock fronts. Nonetheless, the reconnection rates observed in our models are consistent with kinetic simulations (e.g., Shav et al. 1999), and the code still approximately fulfills global energy conservation, thus providing a first description of the net dissipation of magnetic energy into plasma heating. Hence, we believe the simulations presented here represent a physically reasonable scenario for heating by centrifugally driven reconnection, with the associated X-ray emission providing a promising explanation for the X-ray flares observed from magnetic Ap/Bp stars.

\section{SUMMARY}

The rigid-body corotation makes the equatorial disks in our simulations quite distinct from the Keplerian disk envisioned in the MTD scenario promoted by Cassinelli et al. (2002). We find no evidence of an extended region of Keplerian rotation. Instead the results correspond more closely to those derived in the RRM analysis of Townsend \& Owocki (2005), although the confinement parameter we consider is near the lower end of the range of validity for the basic rigid-field approach.

Moreover, our numerical simulations further suggest a novel mechanism for magnetic reconnection, driven by episodic centrifugal breakout events. The strong reconnection heating associated with such breakout represents a promising mechanism for explaining X-ray flares observed in magnetic Bp stars such as $\sigma$ Ori E.

In future work, we plan to use more-extensive numerical tools to investigate in greater detail the reconnection processes within this centrifugally driven breakout scenario. We also plan to explore hybrid methods that could allow extensions of MHD simulations to larger confinement parameters, including 3D models of tilted-field cases more appropriate to $\sigma$ Ori $\mathrm{E}$ and other magnetic hot-stars. 
We thank Michael Shay and an unnamed referee for their helpful comments on the physics of recon- nection. We acknowledge support from NASA grants LTSA/NNG05GC36G and Chandra/GO3-3024C.

\section{REFERENCES}

Babel J., Montmerle T., 1997a, ApJ, 485, L29

Babel J., Montmerle T., 1997b, A\&A, 323, 121

Cassinelli J. P., Brown J. C., Maheswaran M., Miller N. A., Telfer D. C., 2002, ApJ, 578, 951

Donati J.-F., Babel J., Harries T. J., Howarth I. D., Petit P., Semel M., 2002, MNRAS, 333, 55

Feigelson E. D., Broos P., Gaffney J. A., Garmire G., Hillenbrand L. A., Pravdo S. H., Townsley L., Tsuboi Y., 2002, ApJ, 574, 258

Furth H. P., Killen J., Rosenbluth M. N., 1963, Phys. Fluids, 6, 459

Gagné M., Oksala M. E., Cohen D. H., Tonnesen S. K., ud-Doula A., Owocki S. P., Townsend R. H. D., MacFarlane J. J., 2005, ApJ, 628, 986

Groote D., Schmitt J. H. M. M., 2004, A\&A, 418, 235

Jardine M., Collier Cameron A., Donati J.-F., Pointer G. R., 2001, MNRAS, 324, 201

Jardine M., van Ballegooijen A. A., 2005, MNRAS, 361, 1173

MacGregor K. B., 1988, ApJ, 327, 794
Miyagoshi T., Yokoyama T., 2003, ApJ, 593, L133

Nitta S.-y., 2004, ApJ, 610, 1117

Petschek H. E., 1966, in NASA Spec. Publ. 50: AAS-NASA Symposium on Physics of Solar Flares p. 425

Sanz-Forcada J., Franciosini E., Pallavicini R., 2004, A\&A, 421, 715

Shay M. A., Drake J. F., Rogers B. N., Denton R. E., 1999, Geophys. Res. Lett., 26, 2163

Shore S. N., Brown D. N., 1990, ApJ, 365, 665

Stone J. M., Norman M. L., 1992, ApJS, 80, 753

Townsend R. H. D., Owocki S. P., 2005, MNRAS, 357, 251

Townsend R. H. D., Owocki S. P., Groote D., 2005, ApJ, 630, L81 ud-Doula A., 2003, PhD thesis, University of Delaware ud-Doula A., Owocki S. P., 2002, ApJ, 576, 413

Wiechen H., Büchner J., Otto A., 1995, J. Geophys. Res., 100, 19259

Yanagida T., Ezoe Y.-I., Makishima K., 2004, PASJ, 56, 813 